\title{
The Challenges of Data Acquisition in Harsh Remote Places
}

\section{Dave Buckley}

\author{
Curtiss-Wright
}

Dublin, Ireland

\begin{abstract}
In modern flight test installations there is a continuing trend to move the data acquisition closer to the sensors. As a consequence the data acquisition chassis needs to be mounted in locations that are small, inaccessible and subject to harsh environmental conditions. On top of this there are an increasing number of measurements required for each new flight test campaign. This paper discusses the challenges of designing a small lightweight data acquisition chassis which can provide hundreds of channels of measurement capability while operating in tight spaces which are exposed to fluids, high vibration and extremes of temperature. The paper suggests ways of designing and installing the data acquisition chassis in order to optimize the available installation space while mitigating the effects of the harsh environmental conditions.
\end{abstract}

Keywords: Data Acquisition, DAU, Modular, Flexible, Remote, Miniature, IEEE 1588, INET

\section{INTRODUCTION}

In a drive to reduce the wiring of flight test installations there is a continuing trend of moving the data acquisition chassis closer to the sensors. As a consequence the data acquisition chassis needs to be mounted in locations that are small, inaccessible and subject to harsh environmental conditions. This paper discusses the implications of these requirements on the design of the data acquisition chassis. The paper begins with a recap of some of the more important data acquisition design concepts such as reliability and modularity which are so important for flight test equipment. The paper then investigates how small the data acquisition chassis can get while maintaining modularity and flexibility. The paper also presents some solutions to the difficult environmental conditions that are found in remote locations, such as high temperature and exposure to fluids. Finally the paper examines some of the system requirements placed on miniature modular data acquisition chassis. 


\section{DATA ACQUISITION DESIGN CONCEPTS}

Modern data acquisition chassis require a very high degree of flexibility and configurability. Flexibility can be provided on the chassis level by designing each chassis to consist of multiple acquisition cards, with each acquisition card carrying out a different function. Further flexibility can be provided at the card level by allowing the behavior of the acquisition card to be configured. At the chassis level the flight test instrumentation engineer can create almost any configuration with a large catalog of acquisition modules.

Depending on the platform, the size envelope available to install a data acquisition chassis will vary. Therefore it could be argued that the flight test instrumentation engineer requires multiple chassis types, each of which will house different sized data acquisition cards. In this scenario each chassis will come with its own catalog of acquisition cards. However this approach has its disadvantages. Firstly the flight test instrumentation engineer will not be able to mix and match his acquisition cards between different chassis. A card from one chassis will not necessarily fit into a second chassis type. Secondly it is unlikely the vendor of the equipment will support all interfaces in all chassis types. Therefore many possible configurations will not be supported.

In fact it is possible to create many different chassis shapes and sizes using the same sized data acquisition cards. Figure 1 shows many examples of a KAM 500 chassis all of which use the same data acquisition cards. This solution allows you to tailor your chassis for different size envelopes while choosing acquisition cards from a single large catalog.

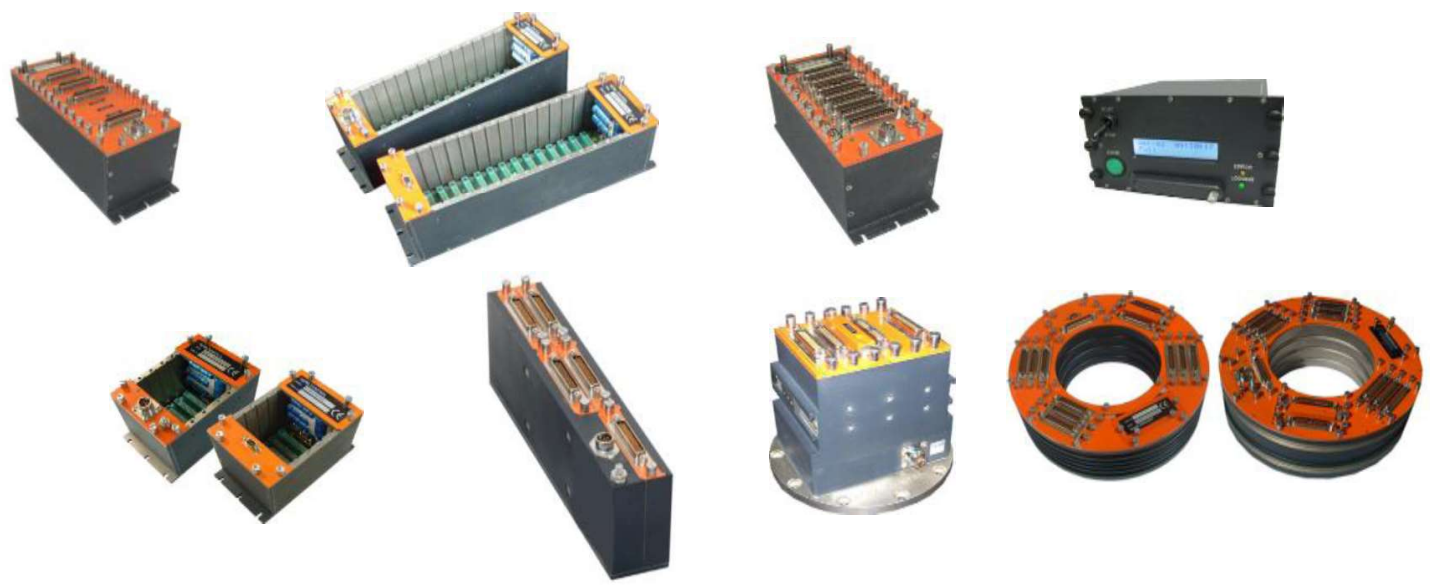

Figure 1: KAM 500 Chassis Shapes and Sizes

All the chassis in Figure 1 are solid chassis in the sense that the there is a chassis into which data acquisition cards are inserted. Another method of building chassis is to construct the chassis out of the acquisition cards themselves. Using this "slice of bread" method there is no separate chassis. The chassis is formed by connecting several acquisition cards together and securing them via some locking mechanism. This method has the advantage that the flight test engineer can build a chassis with any number of slots up to a maximum value. However the "slice of bread approach" also has a number of disadvantages. Firstly when removing a module from the chassis it is not a simple matter of removing the module that you would like to change. The entire chassis must be disassembled in order to remove any module. Secondly the orientation of the modules in 
the chassis cannot change. Using the solid chassis approach shown in Figure 1 the orientation of the acquisition cards in the chassis can changed to create a long narrow chassis or even a circular chassis which could be mounted on a rotor. Furthermore it is also possible with the solid chassis approach to create a chassis which is any number of slots in length, up to a maximum value.

Arguably the most important feature of a data acquisition chassis is reliability. If the acquisition chassis malfunctions during flight then the test points will need to be reflown. This incurs a large expense. It has been shown that designing data acquisition chassis using FPGA based state machines produces extremely reliable data acquisition products. Even if the system gets into an unforeseen state due to power dips during flight it will cycle out of that state within one acquisition cycle and begin operating normally again. It is quite common for processor based systems to not recover fully after such an event. Also in the event of a brief loss of power to the acquisition chassis, a chassis designed using an FPGA based state machine approach will begin acquiring data immediately after resumption of power. This is due to the fact that there are no processors which need to reboot. Acquiring immediately on power up enables test points to be completed even when there is a temporary power interruption to the acquisition system.

\section{MOVING CLOSER TO SENSORS}

It was noted in section 2 that using a solid chassis approach chassis of many different shapes and sizes can be created, all of which use the same catalog of data acquisition cards. However there is a limitation to how small you can make a data acquisition chassis which houses a particular type of data acquisition card. In order to house at least one card the chassis must be larger than the dimensions of a single card. In practice the chassis needs to be significantly bigger than a single acquisition card as the minimum requirement for a chassis would typically be an acquisition card, a transmitter card (to send data via Ethernet or IRIG 106 chapter 4 PCM) and a power supply.

The amount of wiring required on a flight test installation has always been a concern for flight test instrumentation engineers. The time taken to define and install the wiring, the necessity to drill holes through structures and the sheer weight of the wiring bundle are all reasons why there is a continuing drive to reduce the quantity of wiring on a flight test article. One way to reduce the quantity of wiring is to move the data acquisition chassis closer to the sensors. This has the advantage of replacing a section of the wiring loom with a single Ethernet cable from the chassis. As the data acquisition chassis moves closer and closer to the sensors the available locations where a chassis can be installed get smaller. In some cases the space envelope may be smaller (at least in 2 dimensions) than the dimensions of the data acquisition card that is used in the rest of the configuration.

One solution for these locations could be the creation of a dedicated acquisition box which fits in the required dimensions with a small number of measurements. However a dedicated acquisition box will solve the acquisition needs of only one location on one test article. A new box would need to be defined and created for every other location, which 
would typically have a different number and different types of measurements. The way to solve this generally would be to create a miniature modular chassis which could be populated with miniature acquisition cards.

However even this approach has its limitations. As noted previously the smallest modular chassis will typically require an acquisition card, a transmitter and a power supply. As data acquisition chassis get smaller the power supply is increasingly becoming a larger percentage of the volume. This is due to the fact that any piece of equipment which is connected to aircraft power must comply with standards such as MIL STD 704 to ensure that it can be used safely on the aircraft.

In order to fit the acquisition into even smaller spaces it may be necessary to mount the acquisition card itself in a separate location to the chassis. This acquisition card would send its acquired data back to the chassis via a serial cable from which it would also be powered. This would ensure that the acquisition card could fit in a space that was just marginally larger than its own dimensions. Multiple of these remote cards could be connected to single chassis to allow a network of miniature acquisition to be placed in the tightest of spaces. The fact that these cards could be used internal or external to the chassis would allow a relatively large catalog of cards to be created.

\section{ENVIRONMENTAL CONCERNS}

Another consequence of moving the data acquisition chassis closer to the sensors is that the chassis will get placed in more inhospitable places. For example one location for remote chassis is in the engine casing. During some phases of the flight test the ambient temperature of the casing will be in excess of 100 degrees Celsius. The electronics of the acquisition chassis will also add some self-heating. The 2 primary means of removing heat from a chassis are convection via air flow and conduction via the surface that the chassis is installed on. However in some locations there is very little airflow and the surface on which the chassis is installed is not thermally conductive. In this case depending on how much power is being consumed in the chassis, the chassis may be between 20 and 40 degrees hotter than ambient. This can result component temperatures outside the operating range of even military grade components.

One potential solution is to add a large heat sink to the chassis to increase the surface area and allow more heat to be dissipated by convection. However this results in a much bigger chassis, negating the advantage of a small sized chassis, and prevents the chassis from being installed in many of the locations it could have been installed without the heatsink.

Another potential solution is to locate many of the acquisition cards remotely from the chassis. This drastically reduces the heat generated in the chassis itself as most of the power will be consumed by the electronics on the acquisition cards. Also the surface area of each acquisition card would be sufficient to dissipate significantly more heat than if the cards were physically located together in chassis. 
Another inhospitable location for a miniature data acquisition chassis is the landing gear of a fixed wing aircraft. While the temperature will be more benign in these locations the chassis may be more exposed to the elements and sprayed with various fluids while on the ground. One of the challenges with a modular chassis is ensuring that the chassis is fully weather sealed. A chassis that is designed to allow modules to be quickly and easily removed may have small gaps between the modules when they are installed in the chassis. These gaps can be filled using form in place gaskets. Form in place gaskets use elastomer to provide sealing between two surfaces. The elastomer is applied to one side of the acquisition module as shown in Figure 2. When the modules are placed in the chassis side by side, the compression and the cohesion of the elastomeric material will then provide sealing. These gaskets can be electrically conductive and also provide an RFI shield.
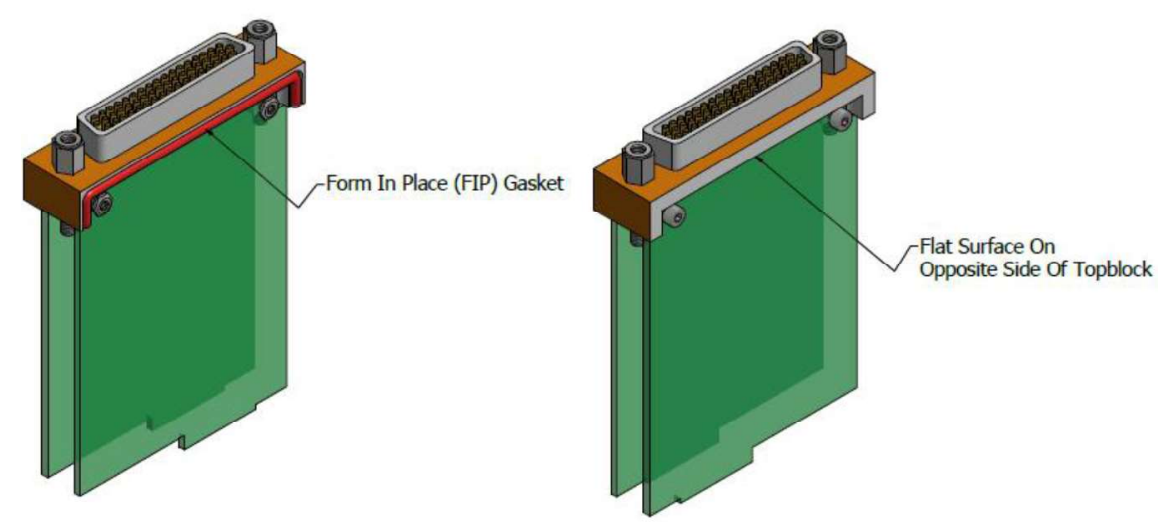

Figure 2: Form in place gaskets

\section{SYSTEM SOLUTIONS}

In section 3 we discussed how the requirement to move the data acquisition chassis ever closer to the sensors could necessitate a miniature acquisition chassis with its own catalog of acquisition modules. However it is important to note that any miniature chassis would need to be fully compatible with existing data acquisition chassis such that a heterogeneous network of standard and miniature chassis could be created. It should be possible to program both chassis types from the same configuration software. The entire configuration should be stored in a single configuration file, for example XidML [1]. It should be possible to analyze the acquired data from both chassis together in the same analysis software. Moreover in order to correlate the parameters from all channels in a heterogeneous network all channels must sample simultaneously. The network synchronization protocol IEEE 1588 [2] can be used to synchronize each chassis such that each chassis has the correct absolute. However it is equally important that both chassis have the same sampling strategy. For example if each chassis samples at the start of an acquisition cycle and at equal intervals thereafter and if the acquisition cycle is tied to absolute time then once the chassis are synchronized via IEEE 1588 they will also sample data simultaneously.

In fact to provide full flexibility of configuration each miniature chassis should be a 
full network node. This would allow any number of the miniature chassis to be added to a network which also included third party equipment. There is a large of number of open standards which can be used on Ethernet networks to ensure interoperability between equipment from different vendors. In particular the INET working group [3] is defining a superset of these standards which should be supported such that there is consistent interface on flight test equipment from all vendors. In order for a miniature data acquisition chassis to be placed in any network, support for these INET standards is an important requirement.

In order to simplify the definition, installation and setup of the network it is also important that the miniature data acquisition chassis communicate with each other via Ethernet. In a typical flight test network a data acquisition chassis will acquire and packetize data, and forward those packets on to a recorder, a telemetry bridge or an on board processor. In modern flight test networks the telemetry bridge, recorder and processor may in fact be housed in one of the data acquisition chassis.

In an Ethernet network multiple nodes are connected via a network switch. The network switch can be a standalone box or a module that fits into the data acquisition chassis. While it is a necessity that a miniature acquisition chassis be able to house a switch module, in some configuration all of the slots in the chassis may be used for acquisition cards. One potential solution to this is to add daisy chaining capability to the chassis. In this scenario the controller card in the chassis would accept an Ethernet input from another chassis and combine the packets it receives from that Ethernet input with its own output packets for transmission upstream. By this method a number of chassis could be installed in tight spaces and connected together without the need for network switches or switch modules. Additionally given that the chassis is an independent network node, third party network equipment could also be daisy chained to the chassis in this way.

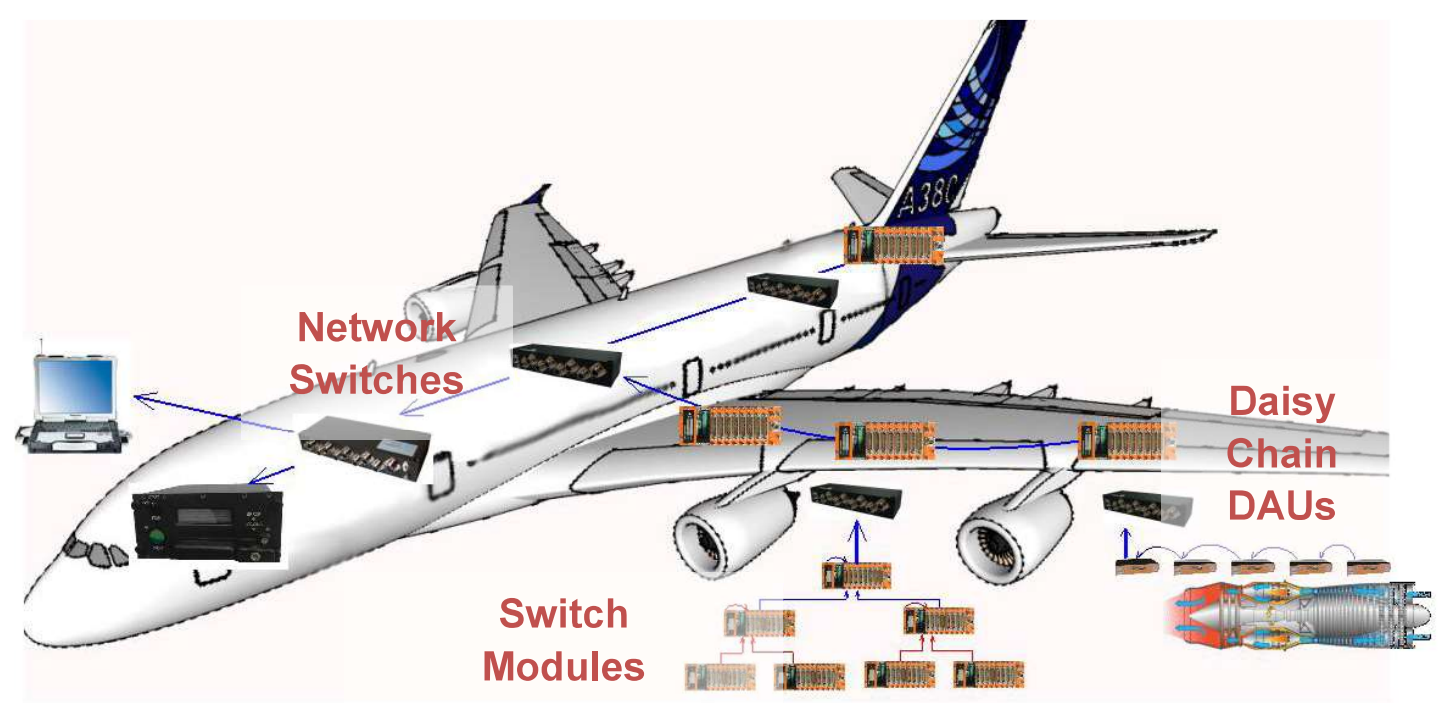

Figure 3: Network with network switches, module switches and daisy chained DAUs 


\section{CONCLUSION}

The trend to move the data acquisition chassis closer to the sensor leads to many challenges for the design of a data acquisition chassis. With the use of a solid chassis approach many different sized data acquisition chassis can be created. This has the advantage that the same acquisition cards can be used in all chassis regardless of whether they are located in the cabin or remotely. However in some cases the small space envelopes available for the installation of the chassis lead to the requirement for a miniature chassis.

A miniature chassis will have similar requirements to the standard chassis in particular modularity and reliability. It has been proven that designing data acquisition chassis using FPGA based state machines produces extremely reliable data acquisition products. One complication of modularity is that there is a limit to how small a miniature modular chassis can be made. One potential solution to this is to locate the acquisition cards remotely from the chassis. This will allow data acquisition solutions to be fit into very small locations.

Locating the data acquisition chassis closer to the sensors can also lead to the chassis being placed in inhospitable places. Placing the chassis in high temperature zones can cause the components to reach temperatures outside of their specification. This can be a particular problem for miniature chassis where a large amount of electronics is squeezed into a small box. Building a chassis with remotely mounted modules may also serve to alleviate this problem. Fluid ingress is another challenge for modular chassis that are installed in locations which are exposed to the elements. However weather sealing can be accomplished using such technologies as form in place gaskets.

Finally it is important that a miniature chassis operates in a heterogeneous network with standard chassis from the same vendor and equipment from third parties. To ensure this, the chassis must be a full network node supporting open standards, including the soon to be published INET standards. In order to connect multiple miniature nodes to the network, it would be a significant advantage if the chassis had the built in capability to daisy chain other network nodes without needing a separate module. With these capabilities the remote miniature chassis would solve many of the challenges flight test engineers are currently facing.

\section{REFERENCES}

[1] A. Cooke, "History and Evolution of Metadata Standards for the FTI," in ITC, San Diego, 2014.

[2] IEEE, Precision clock synchronization protocol for networked measurement and control systems, IEEE Std. 1588, 2008.

[3] J. K. M. M. B. A. T Grace, "key components of the INET test article standard," in ITC, Las Vegas, 2009. 